\title{
MAXIMAL FIELDS DISJOINT FROM CERTAIN SETS
}

\section{P. J. MCCARTHY 1}

Suppose that $C$ is an algebraically closed field and that $Q$ is a subfield of $C$. If $S$ is a nonempty subset of $C$ disjoint from $Q$, it follows from an application of Zorn's lemma that there is a subfield $k$ of $C$ which is maximal with respect to the properties that $Q \subseteq k$ and $k$ and $S$ are disjoint. The problem is to describe the field extension $C / k$. When $S$ consists of a single element this has been done by Quigley [4, Theorems 1, 2 and 3]. In this note we shall give several theorems which describe $C / k$ when $S$ consists of exactly two elements. When $S$ contains more than two elements, some of the arguments used in the proof of Theorem 2 fail.

The first theorem holds when $S$ is any finite (nonempty) subset of $C$ disjoint from $Q$. It generalizes one of Quigley's results [4, Lemma 1].

Theorem 1. If $S$ is a finite set then the extension $C / k$ is algebraic.

Proof. If $C / k$ is transcendental, and if $t$ is an element of $C$ which is transcendental over $k$, then $k(t)$ contains some element of $S$, say $a_{1}$. Then $a_{1}$ is transcendental over $k$, so $a_{1} \notin k\left(a_{1}^{2}\right)$ and $k \neq k\left(a_{1}^{2}\right)$. Hence, $k\left(a_{1}^{2}\right)$ contains some element of $S$, say $a_{2}$, and $a_{2} \neq a_{1}$. Then $a_{2}$ is transcendental over $k$, so $a_{2} \notin k\left(a_{2}^{2}\right)$ and $k \neq k\left(a_{2}^{2}\right)$. Also, $a_{1} \notin k\left(a_{2}^{2}\right)$ since $k\left(a_{2}^{2}\right) \subseteq k\left(a_{1}^{2}\right)$. We repeat this argument until $S$ is exhausted. If $a_{n}$ is the final element of $S$ we have $k \neq k\left(a_{n}^{2}\right) \subseteq \cdots \subseteq k\left(a_{2}^{2}\right) \subseteq k\left(a_{1}^{2}\right)$ and $a_{i} \notin k\left(a_{n}^{2}\right)$ for $i=1, \cdots, n$. This contradicts the defining property of $k$. Hence, $C / k$ must be algebraic.

Henceforth, we assume that $S$ consists of two distinct elements, $a$ and $b$, of $C$. A finite extension $K$ of $k$ will be called cyclic if it is normal over $k$ and if $G(K / k)$, the group of automorphisms of $K$ which leave each element of $k$ fixed, is cyclic. We do not require that $K$ be separable over $k$.

THEOREM 2. There are primes $p$ and $q$ (which may be equal) such that every finite extension of $k$ in $C$ is cyclic of degree $p^{r} q^{*}$ over $k$, for some integers $r$ and $s$.

We consider two cases. In the first case, we suppose that either $a \in k(b)$ or $b \in k(a)$ : to be definite, assume the latter. If $K$ is a proper extension of $k$ in $C$ then either $a \in K$ or $b \in K$, and so we always have

Received by the editors September 17, 1965.

1 Research supported by NSF Grant GP1738. 
$b \in K$. In Quigley's terminology, $k$ is a maximal field without $b$. Thus, in this case, $C / k$ is described by Quigley's results, and the result of the theorem holds.

From now on we shall assume that $a \notin k(b)$ and $b \notin k(a)$. We continue the proof of Theorem 2 with a series of lemmas, the first of which is given in [4].

LEMMA 1. Let $N$ be a finite normal separable extension of a field $F$. Let $p$ be a prime divisor of $[N: F]$. Then there is a sequence of extensions $F \subseteq L_{r} \subset L_{r-1} \subset \cdots \subset L_{0}=N$ such that for $i=1, \cdots, r, L_{i-1} / L_{i}$ is normal of degree $p$, and $p$ does not divide $\left[L_{r}: F\right]$.

Lемма 2. There are primes $p$ and $q$ such that $k(a) / k$ is normal of degree $p$ and $k(b) / k$ is normal of degree $g$.

Proof. Assume that $k$ is perfect. We show first that there is a normal extension of $k$ in $C$ which contains one of $a$ and $b$ but not the other. Assume this is not the case, and let $N$ be the smallest normal extension of $k$ in $C$ which contains $a$. Then $b \in N$ and, in fact, $N$ is the smallest normal extension of $k$ in $C$ which contains $b$. If we use Lemma 1 and the fact that $a \notin k(b)$ and $b \notin k(a)$, we conclude that $[k(a): k]$ and $[k(b): k]$ are relatively prime. Let $p$ be a prime which divides $[k(a): k]$. Since $p$ divides $[N: k]$ it follows from [4, Theorem 6] that there is a maximal subfield $K$ of $C$ without $b$, having exponent $p$, with $k \subseteq K$. Suppose $K \neq k$. Then $a \in K$ and so $k(a) \subseteq K$. By [4, Theorem 2], $[K N: K]$ is a power of $p$, and so the same is true of $[N: K \cap N]$ by the TNI (Theorem of Natural Irrationality [1, p. 149]). Note that $[N: K \cap N] \neq 1$ since $b \notin K$. If $H$ is the subgroup of $G(N / k)$ having $K \cap N$ as its fixed field, then $H$ is a $p$-subgroup of $G(N / k)$. Let $F$ be the fixed field of the Sylow-p-subgroup of $G(N / k)$ which contains $H$. Then $F \subseteq K \cap N$. If $F=k$ then $[N: k]$ is a power of $p$, so $p$ divides $[k(b): k]$, which is not true. Hence $F \neq k$, and since $b \notin F$ we have $k(a) \subseteq F$. But this cannot happen since $p$ does not divide $[F: k]$. Thus, we are forced to conclude that $K=k$. Then, by [4, Theorem 2], $[k(b): k]$ is a power of $p$, again contrary to fact.

Thus, we may assume that there is a normal extension of $k$ in $C$ which contains $a$ but not $b$. An application of Lemma 1 shows that $k(a) / k$ is normal of degree $p$. If $k(b) / k$ is not normal, there is a $k$ automorphism $\sigma$ of $C$ such that $k(\sigma(b)) \neq k(b)$. Then $b \notin k(\sigma(b))$ and so $k(a) \subseteq k(\sigma(b))$. If we apply $\sigma^{-1}$ to $k(\sigma(b))$ and use the fact that $k(a) / k$ is normal, we get $a \in k(b)$, contrary to assumption. Thus, $k(b) / k$ is normal, and we can use Lemma 1 to show that $[k(b): k]=q$ for some prime $q$. 
Now, assume that $k$ is imperfect and let $p$ be the characteristic of $C$. Let $c \in C$ be such that $c \notin k$ but $c^{p} \in k$. Then, $k(c) / k$ is purely inseparable of degree $p$ and so contains exactly one of $a$ and $b$, say $a$. Thus $k(a) / k$ is normal of degree $p$. By the argument used in the preceding paragraph we show that $k(b) / k$ is normal. If $k(b) / k$ is separable it follows from Lemma 1 that $[k(b): k]=q$ for some prime $q$. If $k(b) / k$ is inseparable, then $k\left(b^{p}\right) \neq k(b)\left[1\right.$, p. 130], and since $a \notin k\left(b^{p}\right)$ we must have $b^{p} \in k$. Then $k(b) / k$ is purely inseparable of degree $p$. Actually, this last situation cannot occur. For, if $k(a) / k$ and $k(b) / k$ are both purely inseparable of degree $p$, then so is $k(a+b) / k$ and so either $a \in k(a+b)$ or $b \in k(a+b)$. In the former case $b \in k(a)$, and in the latter $a \in k(b)$, contrary to assumption. This completes the proof of Lemma 2.

The following lemma is proved easily by induction.

LeMmA 3. Let $G$ be a group of order $p^{n}$, where $p$ is a prime and $n \geqq 2$. If $G$ has more than one subgroup of index $p$, then it has at least $p+1$ subgroups of index $p$.

Lemma 4. If $k$ is perfect then $p \neq q$.

Proof. Suppose $p=q$. We use Lemma 1, and the fact that $k(a)$ $\neq k(b)$, to show that if $N$ is the smallest normal extension of $k$ in $C$ which contains both $a$ and $b$, then $[N: k]=p^{n}$ and $n \geqq 2$. Since $k(a)$ and $k(b)$ are the only subfields of $N$ of degree $p$ over $k, G(N / k)$ has exactly two subgroups of index $p$, which contradicts Lemma 3. Thus, $p \neq q$.

To complete the proof of Theorem 2 we show that every finite normal separable extension of $k$ in $C$ is cyclic of degree $p^{r} q^{s}$ for some integers $r$ and $s$. It follows from this, that for a given positive integer $n$, $k$ has at most one separable extension of degree $n$ in $C$. Hence, by [2, Theorem 9], every finite extension of $k$ in $C$ is cyclic. Since every finite extension of $k$ in $C$ has a degree over its separable part equal to some power of the characteristic of $C$, Theorem 2 will follow.

Let $N$ be a finite normal separable extension of $k$ in $C$. If $k$ is imperfect we continue to assume $a$ is inseparable over $k$. Then $a \notin N$, so $b \in N$ and it follows from Lemma 1 that $[N: k]$ is a power of $q=[k(b): k]$. Also, $N$ has exactly one subfield, $k(b)$, of degree $q$ over $k$. Hence, $G(N / k)$ is cyclic [3, Theorem 12.5.3].

Suppose that $k$ is perfect. If $N$ contains only one of $a$ and $b$ we repeat the above argument to show that $N / k$ is cyclic of degree a power of $p$ or a power of $q$. Assume $N$ contains both $a$ and $b$. Then $G=G(N / k)$ has exactly two maximal subgroups, one of index $p$ and 
the other of index $q$ (and $p \neq q$ ). These maximal subgroups are normal in $G$, since $k(a) / k$ and $k(b) / k$ are normal, and so $G$ is nilpotent [3, Corollary 10.3.4]. Hence, $G$ is the direct product of its Sylow subgroups [3, Theorem 10.3.4]. If $G_{p}$ and $G_{q}$ are the Sylow-p-subgroup and Sylow- $q$-subgroup of $G$, respectively, we see by Lemma 1 that $G=G_{p} \times G_{q}$. The fixed field of $G_{p}$ contains exactly one subfield, $k(b)$, of degree $q$ over $k$. Hence, $G_{q}$ is cyclic. Similarly, $G_{p}$ is cyclic. Hence, $G$ is cyclic of degree $p^{r} q^{8}$ for some integers $r$ and $s$. This completes the proof of Theorem 2 .

Suppose $k$ is perfect. Since $p \neq q$ we may assume $p \neq 2$. It follows from [2, Theorem 11] that for each integer $r \geqq 0$ there is an extension of $k$ in $C$ of degree $p^{r}$ over $k$. Furthermore, it follows from what we have proved that there is only one such extension. Call it $k_{r}$. Then $k=k_{0} \subset k_{1}=k(a) \subset k_{2} \subset \cdots$, and we let $k_{\infty}$ be the union of the $k_{r}$. It follows that $k_{\infty}$ is a maximal subfield of $C$ without $b$. We have $k_{r}$ $=\left\{c \mid c \in C\right.$ and $[k(c): k]=p^{t}$ for some $\left.t \leqq r\right\}$. The structure of $C / k_{\infty}$ is given by the first three theorems of [4].

Now, suppose that $k$ is imperfect. As above, we take $k(a) / k$ to be purely inseparable and $k(b) / k$ to be separable. For each in teger $r \geqq 0$ let $k_{r}=k\left(a^{p^{1-r}}\right)$. Then $k=k_{0} \subset k_{1}=k(a) \subset k_{2} \subset \cdots$ and $\left[k_{r}: k\right]=p^{r}$. Furthermore, $k_{r}=\left\{c \mid c \in C\right.$ and $\left.c^{p^{r}} \in k\right\}$. If $k_{\infty}$ is the union of the $k_{r}$, then $k_{\infty}=k^{p^{-\infty}}[1, \mathrm{p} .128]$ and $k_{\infty}$ is a maximal subfield of $C$ without $b$. Again, the structure of $C / k_{\infty}$ is given by theorems in [4].

In both the perfect and imperfect cases we set $K_{r}=\{c \mid c \in C, c$ is separable over $k$, and $[k(c): k]=q^{t}$ for some $\left.t \leqq r\right\}$. Then $K_{r}$ is a subfield of $C$ and $K_{r} \subseteq K_{r+1}$ for all $r$. Let $K_{\infty}$ be the union of the $K_{r}$. It may happen, when $C$ has characteristic zero and $q=2$, that $K_{r}=k(b)$ for all $r \geqq 1$. If this is not the case, then $K_{r} \subset K_{r+1}$ for all $r \geqq 0$.

We can now state the following theorem, which completes our description of $C / k$.

THEOREM 3. Let $L$ be an extension of $k$ in $C$. Then, for some $r$ and $s$, one or both of which may be infinity, we have $L=k_{r} K_{s}$. In this case, $[L: k]=p^{r} q^{s}$.

Proof. If $k$ is perfect we set $E=\{c \mid c \in L$ and $[k(c): k]$ is a power of $p\}$ and $F=\{c \mid c \in L$ and $[k(c): k]$ is a power of $q\}$. If $k$ is imperfect we let $E$ be the fixed field of $G(L / k)$ and $F$ be the separable part of $L / k$. In both cases, $E=k_{r}$ for some $r, F=K_{s}$ for some $s$, and $L=E F$.

Finally, we can use arguments similar to those used in the proofs of the last three theorems of [4] to obtain existence theorems for the various cases that have arisen. 


\section{REFERENCES}

1. N. Bourbaki, Algère, Chapters 4 and 5, Actualités Sci. Ind., No. 1102, Hermann, Paris, 1950.

2. Basil Gordon and E. G. Straus, On the degrees of finite extensions of a field, Proc. Sympos. Pure Math., Vol. 8, pp. 56-65, Amer. Math. Soc., Providence, R. I., 1965.

3. Marshall Hall, The theory of groups, Macmillan, New York, 1959.

4. Frank Quigley, Maximal subfields of an algebraically closed field not containing a given element, Proc. Amer. Math. Soc. 13 (1962), 562-566.

The University of Kansas 